\title{
A SYSTEMATIC APPROACH FOR SOLVING THE GREAT CIRCLE TRACK PROBLEMS BASED ON VECTOR ALGEBRA
}

\author{
Chih-Li Chen, Ph.D.
}

National Taiwan Ocean University, Taiwan

\begin{abstract}
A systematic approach, based on multiple products of the vector algebra (S-VA), is proposed to derive the spherical triangle formulae for solving the great circle track (GCT) problems. Because the mathematical properties of the geometry and algebra are both embedded in the S-VA approach, derivations of the spherical triangle formulae become more understandable and more straightforward as compared with those approaches which use the complex linear combination of a vector basis. In addition, the S-VA approach can handle all given initial conditions for solving the GCT problems simpler, clearer and avoid redundant formulae existing in the conventional approaches. With the technique of transforming the Earth coordinates system of latitudes and longitudes into the Cartesian one and adopting the relative longitude concept, the concise governing equations of the S-VA approach can be easily and directly derived. Owing to the advantage of the S-VA approach, it makes the practical navigator quickly adjust to solve the GCT problems. Based on the S-VA approach, a program namely GCTPro_VA is developed for friendly use of the navigator. Several validation examples are provided to show the S-VA approach is simple and versatile to solve the GCT problems.
\end{abstract}

Keywords: great circle; vector algebra; multiple products; spherical trigonometry

\section{INTRODUCTION}

The great circle arc is the shortest distance between two non-antipodal points on the Earth's surface. Therefore, without considering the wind and tide effects, the most economical route is the great circle track (GCT). As known, a vessel sailing on the GCT in a constantly varying course is impossible and thus, a series of rhumb lines is necessary to be adopted for approximating the GCT in practice $[2,3,9,18]$. Before doing so, the waypoints must be located. However, to determine the waypoints on the GCT, the navigator has to provide some initial conditions. Theoretically, these given initial conditions include: (1) giving the great circle distances to obtain the latitudes and longitudes of the waypoints (hereafter is called "condition 1"); (2) giving the longitudes of the waypoints to yield the latitudes (hereafter is called "condition 2"); (3) giving latitudes of the waypoints to reach the longitudes (hereafter is called "condition 3 "). Then, locating these obtained waypoints on the Mercator chart or ECDIS (Electronic Chart Display and Information System) for every rhumb line sailing is called the great circle sailings in practical navigation $[2,3,9,18]$. For dealing with GCT problems under all initial conditions, a systematic approach to tackle the problems is necessary and becomes one of our research motivations.

Spherical trigonometry (ST) has been used for solving every navigational triangle. Since the Napier's rules was proposed by John Napier (1550-1617), the navigator has adopted the rules in conjunction with the spherical triangle formulae to solve 
the GCT problems. While using the Napier's rules of the rightangled spherical triangles, one needs to take the vertex as the reference point (RP-V). This method has lasted for several centuries and is adopted in the current international maritime education $[2,3,9,12,14,18]$. In addition, because finding the equator crossing point of the GCT is easier than finding the vertex, a method of taking the equator crossing point as the reference point (RP-E) was proposed [4, 7]. Both the RP-V and RP-E have to decide the reference point in advance when solving the GCT problems; they can be categorized as a kind of indirect methods. In contrast to the indirect methods, some researchers took the departure point as the reference point (RP-F) because such a method can directly obtain the waypoint on the GCT. Due to this advantage, Jofeh [13] used a linear equation of the GCT to represent a straight line on the polar gnomonic chart. According to his method, the waypoints are determined only under given condition 2 . Thereafter, the linear combination of a vector basis (VB) to derive the used formula was also proposed $[5,15,16]$. Although the VB was claimed a method of the operation of the vector, it is actually an essence of geometric analysis, which make the derivation of this method hard to understand and results in redundant formulae. In addition, the VB cannot determine the waypoints under all given initial condition. Apart from the $\mathrm{VB}$, a method using multiple products of the vector algebra (VA) appeared lately $[4,6,7]$. A disadvantage of this method is that all given initial conditions cannot be covered when solving the waypoints on the GCT. Therefore, this paper systematically adopts multiple products of the vector algebra (S-VA) to derive the spherical triangle formulae for solving the GCT problems. Advantages of the S-VA approach are that it can handle all given initial conditions more straightforward and avoid redundant formulae existing in the conventional approaches. A comparison of those mentioned methods are listed in Table 1 for convenience.

As compared with the geometric analysis, the dot or cross products of vector algebra are more convenient to describe the relationships between the sides and the angles of the spherical triangle $[11,19]$. Because the S-VA approach transforms the Earth coordinates system of the latitude and longitude into the Cartesian one and adopts the relative longitude concept, the derivation of the spherical triangle formulae becomes simple and clear. In addition, the mathematical properties of the geometry and algebra are both embedded in the S-VA approach; therefore, they are adopted for constructing the theoretical background of the current research. Based on the S-VA approach, a program namely GCTPro_VA is developed for the use of the navigator. The GCTPro_VA with a friendly interface offers a quick and practical tool for the choice of three given initial conditions.

Apart from the current section, the theoretical backgrounds are presented in Section 2. Computation procedures including of all the given initial conditions and its numerical program are illustrated in Section 3. Several validated examples and discussions are given in Section 4. Finally, Section 5 draws concrete conclusions after recasting this research work.

\section{THEORETICAL BACKGROUNDS}

In the spherical geometry, the spherical triangle is the most concise and simplest form for all the geometrical figures. The fundamental structure of the spherical triangle consists in the relationships between the sides (great circle arcs) and angles (dihedral angles). Among the spherical triangle formulae, the two most important laws are the sine law and side cosine law, which are called the "genetic codes" for developing all the spherical triangle formulae [8].

There are two ways to derive the spherical triangle formulae. One is based on the geometry analysis and the other depends on the vector algebra. Theoretically speaking, the most straightforward and understandable operations are using systematic approach with multiple products of the vector algebra (S-VA) $[8,11,19]$. Since the S-VA implies both the mathematical properties of the geometry and algebra, all the spherical triangle formulae can be easily and directly derived. In this regard, three stages are necessary to derive the used spherical triangle formulae and governing equations with respect to the GCT problems. First, the Earth coordinates system of the latitude and longitude is transformed into the Cartesian coordinates system to yield the vector representation. Then, the S-VA is introduced to derive the used spherical triangle formulae. Finally, aimed at the GCT problems, the concise governing equations are yielded by simplifying the related spherical triangle formulae. All symbols used in this article are listed in NOMENCLATURE.

Tab. 1. A comparison of different methods for solving the GCT problem

\begin{tabular}{|c|c|c|c|}
\hline Authors & Category & $\begin{array}{l}\text { Formulae } \\
\text { derivation }\end{array}$ & $\begin{array}{l}\text { Available } \\
\text { condition }\end{array}$ \\
\hline Holm, 1972 & RP-V & ST & 2 \\
\hline Jofeh, 1981 & RP-F & Linear eqn. & 2 \\
\hline Bowditch, 1981; 2002 & $\mathrm{RP}-\mathrm{V}$ & ST & all \\
\hline Keys, 1983 & RP-V & $\mathrm{ST}$ & all \\
\hline Miller et al., 1991 & RP-F & VB & 1 \\
\hline \multirow{2}{*}{ Chen, 2003} & RP-V, RP-E & ST & all \\
\hline & RP-F & VA (GCEM) & 2 and 3 \\
\hline Cutler, 2004 & RP-V & $\mathrm{ST}$ & all \\
\hline \multirow{2}{*}{ Chen et al., 2004} & RP-V & ST & all \\
\hline & RP-F & VA (GCEM) & 2 and 3 \\
\hline $\begin{array}{l}\text { Nastro and Tancredi, } \\
2010\end{array}$ & RP-F & VB & 1 \\
\hline \multirow{2}{*}{ Chen et al., 2014} & RP-E & $\mathrm{ST}$ & all \\
\hline & RP-F & $\mathrm{VA}(\mathrm{COFI})$ & 1 \\
\hline Chen et al., 2015 & RP-F & $\mathrm{VB}(\mathrm{RT})$ & 1 and 2 \\
\hline $\begin{array}{l}\text { Chen, } 2016 \text { (the } \\
\text { current paper) }\end{array}$ & RP-F & S-VA & all \\
\hline
\end{tabular}




\section{COORDINATES TRANSFORMATION}

First, the Earth is treated as a unitary sphere for practical navigation. Then, the Earth coordinates system of the latitude and longitude is transformed into the Cartesian coordinates system. As shown in Fig. 1, the vector of any point $\mathrm{P}$ on the Earth's surface can be represented by the latitude and the

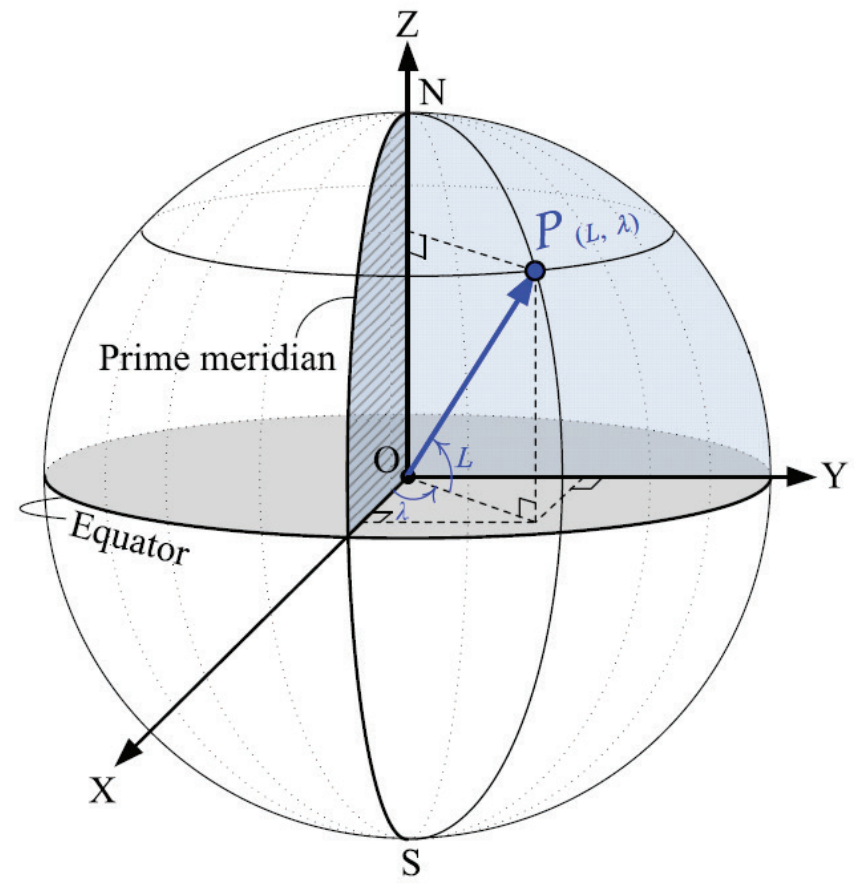

Fig. 1. An illustration of transforming the Earth coordinates system into the Cartesian one

longitude in the Cartesian coordinates system as:

$\overline{\mathbf{P}}=[\cos L \cos \lambda, \cos L \sin \lambda, \sin L], L=\left[-\frac{\pi}{2}, \frac{\pi}{2}\right], \lambda=[0,2 \pi)$.

To avoid an additional judgment of sign convention, the concept of the fixed coordinates system is first considered, that is, the north latitude is treated as a positive value and the south latitude is treated as a negative one. To reduce the number of the variables, the relative longitude concept that replaces the Greenwich meridian by the meridian of the departure point is introduced. As shown in Fig. 2, the unit vectors of the North Pole $(\mathrm{N})$, the departure $(\mathrm{F})$, the destination $(\mathrm{T})$ and the waypoints (X) along the GCT located on the Earth's surface can be expressed as:

$$
\begin{aligned}
& \overrightarrow{\mathbf{N}}=[0,0,1], \\
& \overrightarrow{\mathbf{F}}=\left[\cos L_{\mathrm{F}}, 0, \sin L_{\mathrm{F}}\right],
\end{aligned}
$$

$\overrightarrow{\mathbf{T}}=\left[\cos L_{\mathrm{T}} \cos D L o, \cos L_{\mathrm{T}} \sin D L o, \sin L_{\mathrm{T}}\right]$,
$\overrightarrow{\boldsymbol{X}}=\left[\cos L_{X} \cos D L o_{\mathrm{F} X}, \cos L_{X} \sin D L o_{\mathrm{F} X}, \sin L_{X}\right]$

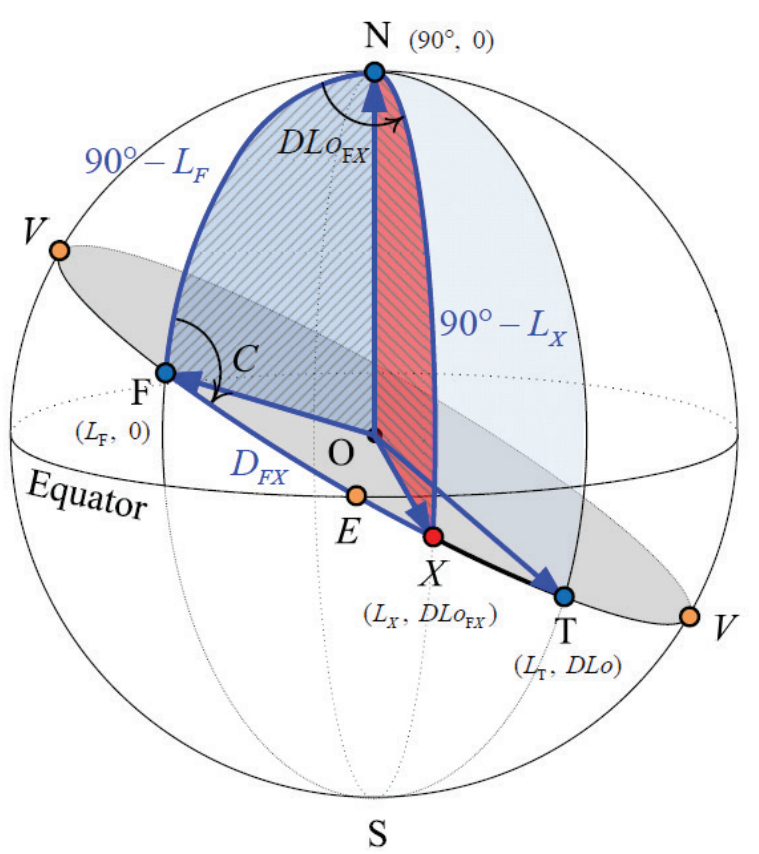

Fig. 2. An illustration of the unit vectors of four points located on the Earth's surface

\section{MULTIPLE PRODUCTS OF VECTOR ALGEBRA}

1. To obtain a great circle arc (side of spherical triangle), we can adopt the dot and cross product of two vectors.

From the viewpoint of geometry, the dot product of two vectors is defined as:

$$
\begin{gathered}
\overrightarrow{\mathbf{N}} \cdot \overrightarrow{\mathbf{F}}=\overrightarrow{\mathbf{F}} \cdot \overrightarrow{\mathbf{N}}=\sin L_{\mathrm{F}}, \overrightarrow{\mathbf{N}} \cdot \overrightarrow{\mathbf{T}}=\sin L_{\mathrm{T}}, \overrightarrow{\mathbf{F}} \bullet \overrightarrow{\mathbf{T}}=\cos D, \\
\overrightarrow{\mathbf{N}} \cdot \overrightarrow{\boldsymbol{X}}=\sin L_{X}, \overrightarrow{\mathbf{F}} \cdot \overrightarrow{\boldsymbol{X}}=\cos D_{\mathrm{F} X}
\end{gathered}
$$

Similarly, the cross product of two vectors is defined as:

$$
\begin{gathered}
|\overrightarrow{\mathbf{N}} \times \overrightarrow{\mathbf{F}}|=|\overrightarrow{\mathbf{F}} \times \overrightarrow{\mathbf{N}}|=\cos L_{\mathrm{F}},|\overrightarrow{\mathbf{N}} \times \overrightarrow{\mathbf{T}}|=\cos L_{\mathrm{T}}, \\
|\overrightarrow{\mathbf{F}} \times \overrightarrow{\mathbf{T}}|=\sin D, \\
|\overrightarrow{\mathbf{N}} \times \overrightarrow{\boldsymbol{X}}|=\cos L_{X},|\overrightarrow{\mathbf{F}} \times \overrightarrow{\boldsymbol{X}}|=\sin D_{\mathrm{F} X} .
\end{gathered}
$$

2. To construct a great circle equation (GCE), we can use the scalar triple products.

A necessary and sufficient condition for the vectors $\overrightarrow{\mathbf{F}}$, $\overrightarrow{\mathbf{T}}$, and $\overrightarrow{\boldsymbol{X}}$ to be coplanar, the volume of the parallelepiped form by three vectors must be zero, that is,

$$
(\overrightarrow{\mathbf{F}} \times \overrightarrow{\mathbf{T}}) \bullet \overrightarrow{\boldsymbol{X}} \equiv(\overrightarrow{\mathbf{F}} \overrightarrow{\mathbf{T}} \overrightarrow{\boldsymbol{X}})=0
$$


Let the coefficient vector be:

$$
(\overrightarrow{\mathbf{F}} \times \overrightarrow{\mathbf{T}})=\left[\mathrm{k}_{1}, \mathrm{k}_{2}, \mathrm{k}_{3}\right]
$$

in which the components of the coefficient vector are as follows:

$$
\begin{gathered}
\mathrm{k}_{1}=-\sin L_{\mathrm{F}} \cos L_{\mathrm{T}} \sin D L o, \\
\mathrm{k}_{2}=\sin L_{\mathrm{F}} \cos L_{\mathrm{T}} \cos D L o-\cos L_{\mathrm{F}} \sin L_{\mathrm{T}}, \\
\mathrm{k}_{3}=\cos L_{\mathrm{F}} \cos L_{\mathrm{T}} \sin D L o .
\end{gathered}
$$

Substituting equations (6a), (6b) and (6c) into equation (5) yields the great circle equation (GCE) as $[4,6,7]$ :

$$
\mathrm{k}_{1} \cos L_{X} \cos D L o_{\mathrm{F} X}+\mathrm{k}_{2} \cos L_{X} \sin D L o_{\mathrm{F} X}+\mathrm{k}_{3} \sin L_{X}=0 .
$$

Equation (7) has two variables, that is $L_{x}$, and $D L o_{F X}$; therefore, we can rearrange equation (7) to obtain two forms in the following. One is

$$
\tan L_{X}=\frac{\mathrm{k}_{1} \cos D L o_{\mathrm{F} X}+\mathrm{k}_{2} \sin D L o_{\mathrm{F} X}}{-\mathrm{k}_{3}},
$$

which can be used to yield latitudes of the waypoints by giving their longitudes.

When we assume

$$
\sin \alpha=\frac{\mathrm{k}_{1}}{\sqrt{\mathrm{k}_{1}^{2}+\mathrm{k}_{2}^{2}}}, \cos \alpha=\frac{\mathrm{k}_{2}}{\sqrt{\mathrm{k}_{1}^{2}+\mathrm{k}_{2}^{2}}},
$$

and substituting them into equation (7), the other form can be yielded as:

$$
\begin{aligned}
\tan \alpha & =\frac{\mathrm{k}_{1}}{\mathrm{k}_{2}}, \\
\sin \left(D L o_{\mathrm{F} X}+\alpha\right) & =\frac{-\mathrm{k}_{3}}{\sqrt{\mathrm{k}_{1}{ }^{2}+\mathrm{k}_{2}{ }^{2}}} \tan L_{X} .
\end{aligned}
$$

The above two equations are a set governing equations. They can be used to obtain longitudes of the waypoints by giving their latitudes.

3. To obtain a dihedral angle (angle of spherical triangle), we can adopt the dot and cross products of two normal vectors of two planes. Therefore the sine law, the side cosine law and the four-part formula can be yielded in the following:

1. Derive the sine law of the spherical triangle by using the cross product of two normal vectors of two planes To obtain the dihedral angle $(C)$, we have

$$
\begin{aligned}
(\overrightarrow{\mathbf{F}} \times \overrightarrow{\mathbf{N}}) \times(\overrightarrow{\mathbf{F}} \times \overrightarrow{\mathbf{X}})= & \left(\cos L_{\mathrm{F}} \sin D_{\mathrm{F} X} \sin C\right) \overrightarrow{\mathbf{F}} \\
& (\overrightarrow{\mathbf{F}} \overrightarrow{\mathbf{N}} \overrightarrow{\mathbf{X}}) \overrightarrow{\mathbf{F}}
\end{aligned}
$$

in which the first row of the above equation is the definition of geometry and the second row is the operation result of vector algebra [11, 19]. Then, from equation (10a), we have

$$
\cos L_{\mathrm{F}} \sin D_{\mathrm{F} X} \sin C=(\overrightarrow{\mathbf{F} \mathbf{N} \mathbf{X}}) .
$$

Similarly, to obtain the dihedral angle $\left(D L o_{F X}\right)$, we have

$$
\begin{aligned}
(\overrightarrow{\mathbf{N}} \times \overrightarrow{\mathbf{X}}) \times(\overrightarrow{\mathbf{N}} \times \overrightarrow{\mathbf{F}}) & =\left(\cos L_{X} \cos L_{\mathrm{F}} \sin D L o_{\mathrm{F} X}\right) \overrightarrow{\mathbf{N}} \\
& =(\overrightarrow{\mathbf{N}} \overrightarrow{\mathbf{X}} \overrightarrow{\mathbf{F}}) \overrightarrow{\mathbf{N}}
\end{aligned}
$$

in which the first row of the above equation is the definition of geometry and the second row is the operation result of vector algebra $[11,19]$. Then, we have:

$$
\cos L_{X} \cos L_{\mathrm{F}} \sin D L o_{\mathrm{F} X}=(\overrightarrow{\mathbf{N X F}}) .
$$

Since the right-hand sides of equations of (10b) and (11b) are equal, we achieve:

$$
\sin D_{\mathrm{F} X} \sin C=\cos L_{X} \sin D L o_{\mathrm{F} X},
$$

which is the sine law of the spherical triangle.

2. Derive the side cosine law of the spherical triangle by using the dot product of two normal vectors of two planes

To obtain the dihedral angle $(C)$, we have:

$$
\begin{aligned}
(\overrightarrow{\mathbf{F}} \times \overrightarrow{\mathbf{N}}) \bullet(\overrightarrow{\mathbf{F}} \times \overrightarrow{\mathbf{X}})=\cos L_{\mathrm{F}} \sin D_{\mathrm{F} X} \cos C \\
\quad=\left|\begin{array}{ll}
(\overrightarrow{\mathbf{F}} \bullet \overrightarrow{\mathbf{F}}) & (\overrightarrow{\mathbf{F}} \bullet \overrightarrow{\mathbf{X}}) \\
(\overrightarrow{\mathbf{N}} \bullet \overrightarrow{\mathbf{F}}) & (\overrightarrow{\mathbf{N}} \bullet \overrightarrow{\mathbf{X}})
\end{array}\right|=\sin L_{X}-\sin L_{\mathrm{F}} \cos D_{\mathrm{F} X},
\end{aligned}
$$

in which the first row of the above equation is the definition of geometry and the second row is the operation result of vector algebra $[11,19]$. Then, from equation (13a), we have:

$$
\cos L_{\mathrm{F}} \sin D_{\mathrm{F} X} \cos C=\sin L_{X}-\sin L_{\mathrm{F}} \cos D_{\mathrm{F} X} .
$$

Similarly, to obtain the dihedral angle $\left(D L o_{F X)}\right.$, we have:

$$
\begin{aligned}
& (\overrightarrow{\mathbf{N}} \times \overrightarrow{\mathbf{X}}) \bullet(\overrightarrow{\mathbf{N}} \times \overrightarrow{\mathbf{F}})=\cos L_{X} \cos L_{\mathrm{F}} \cos D L o_{\mathrm{F} X} \\
& =\left|\begin{array}{ll}
(\overrightarrow{\mathbf{N}} \bullet \overrightarrow{\mathbf{N}}) & (\overrightarrow{\mathbf{N}} \bullet \overrightarrow{\mathbf{F}}) \\
(\overrightarrow{\mathbf{X}} \bullet \overrightarrow{\mathbf{N}}) & (\overrightarrow{\mathrm{X}} \bullet \overrightarrow{\mathbf{F}})
\end{array}\right|=\cos D_{\mathrm{F} X}-\sin L_{\mathrm{F}} \sin L_{X},
\end{aligned}
$$


in which the first row of the above equation is the definition of geometry and the second row is the operation result of vector algebra $[11,19]$. Then, we have:

$$
\cos L_{X} \cos L_{\mathrm{F}} \cos D L o_{\mathrm{F} X}=\cos D_{\mathrm{F} X}-\sin L_{\mathrm{F}} \sin L_{X} \text {. }
$$

It should be noticed that both equation (13b) and equation (14b) are the side cosine laws.

3. Derive the four-part formula of the spherical triangle by using a combination of the sine and side cosine laws

Dividing equations (10a) by equation (13a) yields:

$$
\begin{gathered}
\frac{(\overrightarrow{\mathbf{F}} \times \overrightarrow{\mathbf{N}}) \times(\overrightarrow{\mathbf{F}} \times \overrightarrow{\mathbf{X}})}{(\overrightarrow{\mathbf{F}} \times \overrightarrow{\mathbf{N}}) \bullet(\overrightarrow{\mathbf{F}} \times \overrightarrow{\mathbf{X}})}=\tan C \\
=\frac{\cos L_{\mathrm{F}} \sin D_{\mathrm{F} X} \sin C}{\sin L_{X}-\sin L_{\mathrm{F}} \cos D_{\mathrm{F} X}} .
\end{gathered}
$$

From the above equation, we have:

$$
\tan C=\frac{\cos L_{\mathrm{F}} \sin D_{\mathrm{F} X} \sin C}{\sin L_{X}-\sin L_{\mathrm{F}} \cos D_{\mathrm{F} X}} .
$$

To yield the four-part formula, we have to substitute equations (12) and (14b) into equation (15b) for eliminating $D_{F X}$ Then, we have the four-part formula as:

$\cos L_{\mathrm{F}} \tan C \tan L_{X}=\sin D L o_{\mathrm{F} X}+\sin L_{\mathrm{F}} \tan C \cos D L o_{\mathrm{F} X}$.

\section{DERIVATIONS OF THE GOVERNING EQUATIONS}

1. The great circle distance

There are two ways to yield the great circle distance. One is:

$$
\begin{aligned}
& \overrightarrow{\mathbf{F}} \bullet \overrightarrow{\mathbf{T}}=\cos D \\
& =\cos L_{\mathrm{F}} \cos L_{\mathrm{T}} \cos D L o+\sin L_{\mathrm{F}} \sin L_{\mathrm{T}} .
\end{aligned}
$$

The other is to replace $\overrightarrow{\boldsymbol{X}}$ by $\overrightarrow{\mathbf{T}}$ in the equation (14a). Then, we have:

$$
\begin{aligned}
& (\overrightarrow{\mathbf{N}} \times \overrightarrow{\mathbf{T}}) \bullet(\overrightarrow{\mathbf{N}} \times \overrightarrow{\mathbf{F}})=\cos L_{\mathrm{T}} \cos L_{\mathrm{F}} \cos D L o \\
& =\left|\begin{array}{ll}
(\overrightarrow{\mathrm{N}} \bullet \overrightarrow{\mathbf{N}}) & (\overrightarrow{\mathbf{N}} \bullet \overrightarrow{\mathbf{F}}) \\
(\overrightarrow{\mathbf{T}} \bullet \overrightarrow{\mathrm{N}}) & (\overrightarrow{\mathrm{T}} \bullet \overrightarrow{\mathbf{F}})
\end{array}\right|=\cos D-\sin L_{\mathrm{F}} \sin L_{\mathrm{T}}
\end{aligned}
$$

It is found that equations (17a) and (17b) are the same. Rewriting the equation (17a) yields:

$$
\cos D=\sin L_{\mathrm{F}} \sin L_{\mathrm{T}}+\cos L_{\mathrm{F}} \cos L_{\mathrm{T}} \cos D L o .
$$

It should be noticed that equation (18) is the governing equation to obtain the great circle distance.

2. The great circle initial course angle

There are also two ways to yield the great circle initial course angle. One is to rearrange equation (12), which is the sine law of the spherical triangle. Then, we have:

$$
\sin C=\frac{\cos L_{\mathrm{T}} \sin D L o}{\sin D} .
$$

The other is to replace $\overrightarrow{\boldsymbol{X}}$ by $\overrightarrow{\mathbf{T}}$ in equation (13a), which is the side cosine law of the spherical triangle. Then, we have:

$$
\begin{aligned}
& (\overrightarrow{\mathbf{F}} \times \overrightarrow{\mathbf{N}}) \bullet(\overrightarrow{\mathbf{F}} \times \overrightarrow{\mathbf{T}})=\cos L_{\mathrm{F}} \sin D \cos C \\
& =\left|\begin{array}{ll}
(\overrightarrow{\mathrm{F}} \bullet \overrightarrow{\mathbf{F}}) & (\overrightarrow{\mathrm{F}} \bullet \overrightarrow{\mathbf{T}}) \\
(\overrightarrow{\mathbf{N}} \bullet \overrightarrow{\mathrm{F}}) & (\overrightarrow{\mathrm{N}} \bullet \overrightarrow{\mathrm{T}})
\end{array}\right|=\sin L_{\mathrm{T}}-\sin L_{\mathrm{F}} \cos D .
\end{aligned}
$$

Either equation (19) or equation (20a) can be used to yield the great circle initial course angle; however, for avoiding an additional sign judgment, we suggest the navigator use equation (20a). Rearranging equation (20a) obtains:

$$
\cos C=\frac{\sin L_{\mathrm{T}}-\sin L_{\mathrm{F}} \cos D}{\cos L_{\mathrm{F}} \sin D} .
$$

It should be noticed that equation (20b) is the governing equation to obtain the great circle initial course angle.

3. The waypoints on the GCT

Condition 1: Giving the great circle distances to obtain the latitudes and the longitudes of the waypoints on the GCT

Rearranging equations (13b) and (14b), respectively yields:

$$
\sin L_{X}=\sin L_{\mathrm{F}} \cos D_{\mathrm{F} X}+\cos L_{\mathrm{F}} \sin D_{\mathrm{F} X} \cos C,
$$

and

$$
\cos D L o_{\mathrm{F} X}=\frac{\cos D_{\mathrm{F} X}-\sin L_{\mathrm{F}} \sin L_{X}}{\cos L_{\mathrm{F}} \cos L_{X}} .
$$

It should be noticed that equations (21) and (22) are a set of the governing equations to obtain the latitudes and the longitudes of the waypoints on the GCT under condition 1.

Condition 2: Giving the longitudes of the waypoints to obtain their latitudes

There are two ways to obtain the latitudes of the waypoints by giving the longitudes. Either rearranging equation (16) or substituting equations (18), (19), and (20b) into equation (8) to eliminate DLo yields the same result, which can be expressed as:

$$
\tan L_{X}=\frac{\cos C \sin D L o_{\mathrm{F} X}+\sin L_{\mathrm{F}} \sin C \cos D L o_{\mathrm{F} X}}{\cos L_{\mathrm{F}} \sin C} .
$$


It is noticed, that equation (23a) is the governing equation to yield latitudes of the waypoints on the GCT under condition 2. However, this equation needs to find the great circle initial course angle $(C)$ in advance. To avoid this unnecessary step, we first rearrange equation (16) to obtain:

$$
\tan L_{X} \cos L_{\mathrm{F}}=\cot C \sin D L o_{\mathrm{F} X}+\sin L_{\mathrm{F}} \cos D L o_{\mathrm{F} X} .
$$

Then replacing $\mathrm{X}$ by $\mathrm{T}$ in equation (23b) yields:

$$
\tan L_{\mathrm{T}} \cos L_{\mathrm{F}}=\cot C \sin D L o+\sin L_{\mathrm{F}} \cos D L o .
$$

Finally, substituting equation (23c) into equation (23b) can eliminate $\mathrm{C}$ and introducing additional formula of trigonometric functions to obtain:

$$
\tan L_{X}=\frac{\tan L_{\mathrm{F}} \sin \left(\lambda_{X^{-}} \lambda_{\mathrm{T}}\right)+\sin L_{\mathrm{T}} \sin \left(\lambda_{X^{-}} \lambda_{\mathrm{F}}\right)}{\sin \left(\lambda_{\mathrm{T}^{-}} \lambda_{\mathrm{F}}\right)} .
$$

It is noticed, that equation (23d) is the most concise form of the governing equation (or so called meridian method) to yield latitudes of the waypoints on the GCT under condition 2 [18].

Condition 3: Giving the latitudes of the waypoints to obtain their longitudes

There are also two ways to obtain the longitudes of the waypoints by giving the latitudes. One way is to use a set of governing equations, equations (9a) and (9b), which are derived from the GCE. The other one is yielded from the four-part formula of spherical triangle, that is, equation (16) and derived as follows. First we set

$$
\tan \alpha=\sin L_{\mathrm{F}} \tan C
$$

and then, introduce the additional formula of trigonometric functions into equation (16). Therefore, we have:

$$
\sin \left(D L o_{\mathrm{F} X}+\alpha\right)=\left(\cos \alpha \cos L_{\mathrm{F}} \tan C\right) \tan L_{X}
$$

It should be noticed, that equations (24a) and (24b) are a set of the most concise form of the governing equations to yield longitudes of the waypoints on the GCT under condition 3.

4. The equator crossing point

The latitude of the equator crossing point must be zero. Substituting it into equation (23) and rearranging it can yield the equation:

$$
\tan D L o_{\mathrm{FE}}=-\sin L_{\mathrm{F}} \tan C .
$$

It should be noticed, that equation (25) is the governing equation to obtain the longitude of the equator crossing point.

5. The vertex

The vertices of a great circle are the points nearest the poles. Accordingly, let the first derivative of equation (23) be zero and rearrange it, we get equation:

$$
\tan D L o_{\mathrm{F} V}=\frac{1}{\sin L_{\mathrm{F}} \tan C} .
$$

While using the sine law of the right-angled spherical triangles, we have:

$$
\cos L_{V}=\cos L_{\mathrm{F}} \sin C
$$

It is noticed that equations (26a) and (26b) are a set of the governing equations to yield the vertex.

\section{COMPUTATION PROCEDURES AND NUMERICAL PROGRAM}

As mentioned above, the main task of the GCT problems is to obtain the waypoints on the GCT. These waypoints are then transferred to a Mercator chart or the ECDIS. Then, the course and distance of the rhumb line between two adjacent waypoints are determined by using the spherical Mercator sailing. In this regard, we first introduce all needed formulae used for the spherical Mercator sailing. Thereafter, all the governing equations derived for solving the GCT problems are summarized in computation procedures. Finally, a program GCTPro_VA is developed for navigator friendly use.

\section{THE COURSES AND DISTANCES OF THE RHUMB LINES}

Basically, there are two kinds of sailings to obtain the course and distance of the rhumb line between two adjacent waypoints on the GCT. One is the spheroid rhumb line sailing and the other is the spherical Mercator sailing $[1,3,5,7,17,18]$. Since the spherical Mercator sailing meets most of the needs of navigational practice and the distance for a rhumb line on the navigation sphere is within $0.5 \%$ of the distance on the rhumb line on the spheroid [10], we adopt the spherical Mercator sailing to yield the course and distance of the rhumb line. For convenience, all needed formulae and related constants in the numerical program are listed as follows $[3,7,17,18]$ :

$$
M=a_{e} \ln \left[\tan \left(45^{\circ}+\frac{L}{2}\right) \times\left(\frac{1-e \sin L}{1+e \sin L}\right)^{\frac{e}{2}}\right],
$$

in which nautical miles (nm) [3] $e=0.081819190842622$, for WGS-84 [17]. And then,

$$
\begin{gathered}
l=60^{\prime} \times\left(L_{X_{i+1}}-L_{X_{i}}\right), \text { dlo }=60^{\prime} \times\left(\lambda_{X_{i+1}}-\lambda_{X_{i}}\right), \\
\Delta M=M_{X_{i+1}}-M_{X_{i}}, \\
\tan c m=\frac{d l o}{\Delta M},
\end{gathered}
$$




$$
d m=\left\{\begin{array}{ll}
l \mathrm{sec} c m & , \mathrm{~cm} \neq 90^{\circ} \\
\text { dlocos } L_{X_{i}}, \mathrm{~cm}=90^{\circ}
\end{array} .\right.
$$

\section{CONSTRUCTING COMPUTATION PROCEDURES}

To completely illustrate the derived governing equations used in each step of the computation procedures, they are summarized as follows.

Step 1. Calculating the great circle information:

The great circle distance and initial course angle are yielded by using equations (18) and (20b), respectively.

The equator crossing points of the great circle are obtained by using equation (25).

The vertices of the great circle are reached by using equations (26a) and (26b).

Step 2. Calculating the waypoints on the GCT under different given initial conditions:

Condition 1: Giving the great circle distances to obtain the latitudes and the longitudes of the waypoints on the GCT. The latitudes and longitudes of the waypoints are calculated by using equations (21) and (22), respectively.

Condition 2: Giving the longitudes of the waypoints to yield the latitudes. The latitudes of the waypoints are calculated by using equation (23d).

Condition 3: Giving the latitudes of the waypoints to reach the longitudes. The longitudes of the waypoints are calculated by using equations (24a) and (24b).

Step 3. Calculating the course and distance between two adjacent waypoints: The Mercator course and distance of every rhumb line between two adjacent waypoints are calculated by using equations (27), (28), (29) and (30) of spherical Mercator sailings.

\section{DEVELOPING THE NUMERICAL PROGRAM}

To deal with GCT problems under all given initial conditions, a program, namely GCTPro_VA, is developed based on the S-VA approach. In addition, for selecting the reasonable number of the waypoints, a diagram of total Mercator distance versus waypoints number (hereafter is called tMd-n diagram) is provided for the navigator. The GCTPro_VA program is coded in the Visual Basic 6.0 (VB6) with the graphical user interface (GUI) for popular and friendly use of the navigator.

\section{VALIDATED EXAMPLES AND DISCUSSIONS}

Example 1. A vessel is proceeding from San Francisco (USA) to Sydney (AUSTRALIA). The navigator desires to use great circle sailing from $L 37^{\circ} 47.5^{\prime} \mathrm{N}, \lambda 122^{\circ} 27.8^{\prime} \mathrm{W}$ to L33 ${ }^{\circ} 51.7^{\prime} \mathrm{S}, \lambda 151^{\circ} 12.7^{\prime} \mathrm{E}$ (Bowditch, 1981, pp. 616-618).

Required: Using the GCTPro_VA program to calculate the great circle information and the latitudes and longitudes of the waypoints along the GCT at equal interval of great circle distance, $360 \mathrm{~nm}\left(6^{\circ}\right)$ apart from the departure (Condition 1). In addition, the relationships of total Mercator distance and numbers of the waypoints are acquired.

Solution: The GCTPro_VA program is run to solve the great circle information and the waypoints on the GCT under

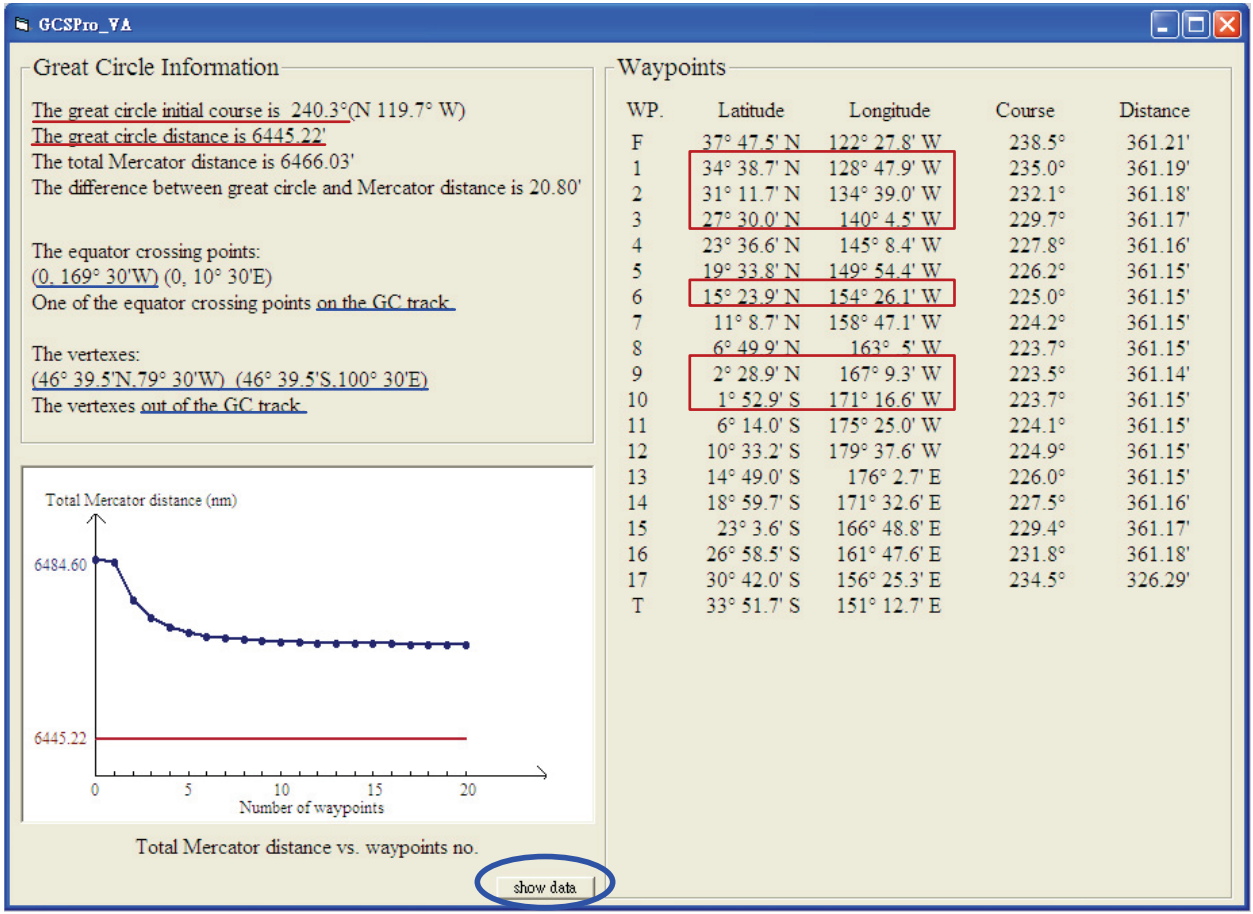

Fig. 3. Results of condition 1 by running the GCTPro_VA program in example 1 
condition 1 successfully. Results of the great circle information, the waypoints on the GCT and a tMd-n diagram are all shown in Fig. 3. The comparison of results obtained by the S-VA approach and those by tabular method (Ageton method) [2] is shown in Table 2. In addition, when the button of "show data" in Fig. 3 is clicked, the relationships between the total Mercator distance and numbers of the waypoints on the GCT will be shown in Table 3 .

Remark: With respect to the waypoints on the GCT under condition 1 , the S-VA approach has been validated by this example. It is noticed that the computerized solution is always more accurate than the tabular method because it is free of rounding errors, which was also reported in Bowditch book [3]. As shown in Table 3 the total Mercator distance of 16 waypoints is nearly equal to that of 8 waypoints and their difference of the distance is less than $1 \mathrm{~nm}$. A reasonable number of waypoints can be considered from this table for the navigator. It is an interesting discovery here.

Example 2. A vessel is proceeding from Sydney (AUSTRALIA) to Balboa (PANAMA). The master desires to use the great circle sailing from $L 33^{\circ} 51.5^{\prime} \mathrm{S}, \lambda 151^{\circ} 13.0^{\prime} \mathrm{E}$ to $L 08^{\circ} 53.0^{\prime} \mathrm{N}, \lambda 079^{\circ} 31.0^{\prime} \mathrm{W}$ (Chen et al, 2004, pp. 317-319).

\section{Required:}

1. Using the GCTPro_VA program to calculate the great circle information and the latitudes and longitudes of the waypoints along the GCT at longitude $170^{\circ} \mathrm{E}$ and

Tab. 2. A comparison of results obtained by tabular method and those by the S-VA approach in example 1

\begin{tabular}{|c|c|c|c|}
\hline \multicolumn{2}{|c|}{ Item } & Tabular method* & GCTPro_VA \\
\hline \multicolumn{2}{|r|}{$D$} & $6445.5^{\prime}$ & $6445.22^{\prime}$ \\
\hline \multicolumn{2}{|r|}{$C$} & $240^{\circ} 17.5^{\prime}$ & $240.3^{\circ}$ \\
\hline \multicolumn{2}{|r|}{$\mathrm{V}$} & $L 46^{\circ} 39.5^{\prime} \mathrm{S}, \lambda 100^{\circ} 29.7^{\prime} \mathrm{E}$ & $\begin{array}{l}L 46^{\circ} 39.5^{\prime} \mathrm{S}, \lambda 100^{\circ} 30.0^{\prime} \mathrm{E} \\
L 46^{\circ} 39.5^{\prime} \mathrm{N}, \lambda 79^{\circ} 30.0^{\prime} \mathrm{W}\end{array}$ \\
\hline \multicolumn{2}{|r|}{$\mathrm{E}$} & - & $\begin{array}{l}L 00^{\circ} 00.0^{\prime}, \lambda 169^{\circ} 30.0^{\prime} \mathrm{W} \\
L 00^{\circ} 00.0^{\prime}, \lambda 010^{\circ} 30.0^{\prime} \mathrm{E}\end{array}$ \\
\hline \multirow{6}{*}{$D_{\mathrm{F} X}$} & $6^{\circ}$ & $L 34^{\circ} 39.0^{\prime} \mathrm{N}, \lambda 128^{\circ} 48.3^{\prime} \mathrm{W}$ & $L 34^{\circ} 38.7^{\prime} \mathrm{N}, \lambda 128^{\circ} 47.9^{\prime} \mathrm{W}$ \\
\hline & $12^{\circ}$ & $L 31^{\circ} 12.0^{\prime} \mathrm{N}, \lambda 134^{\circ} 39.3^{\prime} \mathrm{W}$ & $L 31^{\circ} 11.7^{\prime} \mathrm{N}, \lambda 134^{\circ} 39.0^{\prime} \mathrm{W}$ \\
\hline & $18^{\circ}$ & $L 27^{\circ} 30.0^{\prime} \mathrm{N}, \lambda 140^{\circ} 04.3^{\prime} \mathrm{W}$ & $L 27^{\circ} 30.0^{\prime} \mathrm{N}, \lambda 140^{\circ} 04.5^{\prime} \mathrm{W}$ \\
\hline & $36^{\circ}$ & $L 15^{\circ} 24.0^{\prime} \mathrm{N}, \lambda 154^{\circ} 26.3^{\prime} \mathrm{W}$ & $L 15^{\circ} 23.9^{\prime} \mathrm{N}, \lambda 154^{\circ} 26.1^{\prime} \mathrm{W}$ \\
\hline & $54^{\circ}$ & $L 02^{\circ} 29.0^{\prime} \mathrm{N}, \lambda 167^{\circ} 09.3^{\prime} \mathrm{W}$ & $L 02^{\circ} 28.9^{\prime} \mathrm{N}, \lambda 167^{\circ} 09.3^{\prime} \mathrm{W}$ \\
\hline & $60^{\circ}$ & $L 01^{\circ} 52.5^{\prime} \mathrm{S}, \lambda 171^{\circ} 17.3^{\prime} \mathrm{W}$ & $L 01^{\circ} 52.9^{\prime} \mathrm{S}, \lambda 171^{\circ} 16.6^{\prime} \mathrm{W}$ \\
\hline
\end{tabular}

*Resource : Bowditch, 1981, pp. 616-618 (Ageton method). at each 20 degrees of longitude thereafter to longitude $90^{\circ} \mathrm{W}$ (Condition 2).

2. Using the GCTPro_VA program to calculate the great circle information and the latitudes and longitudes of the waypoints on the GCT at latitude $35^{\circ} \mathrm{S}$ and at each 10 degrees of latitude northward to latitude $05^{\circ} \mathrm{N}$ (Condition 3).

Solution:

1. The GCTPro_VA program is used to solve the great circle information and the waypoints on the GCT under condition 2 successfully. Results of the great circle information, the waypoints on the GCT and a tMd-n diagram are all shown in Fig. 4.

2. The GCTPro_VA program is used to solve the great circle information and the waypoints on the GCT under condition 3 successfully. Results of the great circle information, the waypoints on the GCT and a tMd-n diagram are all shown in Fig. 5.

Remark:

1. With respect to the waypoints on the GCT under conditions 2 or 3 , the S-VA approach has been validated by this example. A comparison of the results of the GCE method [6] and those of the S-VA approach shows that the proposed approach is as accurate as the GCE method. However, the derived governing equations of the S-VA approach are simpler and clearer than those of the GCE method.

2. The developed GCTPro_VA program has also been verified by examples 1 and 2 . It is found, that the GCTPro_VA program shows the advantages of completeness and wide applications for navigator.

\section{CONCLUSIONS}

The proposed S-VA approach has systematically derived the spherical triangle formulae for solving the GCT problems and it can handle the waypoints on the GCT 


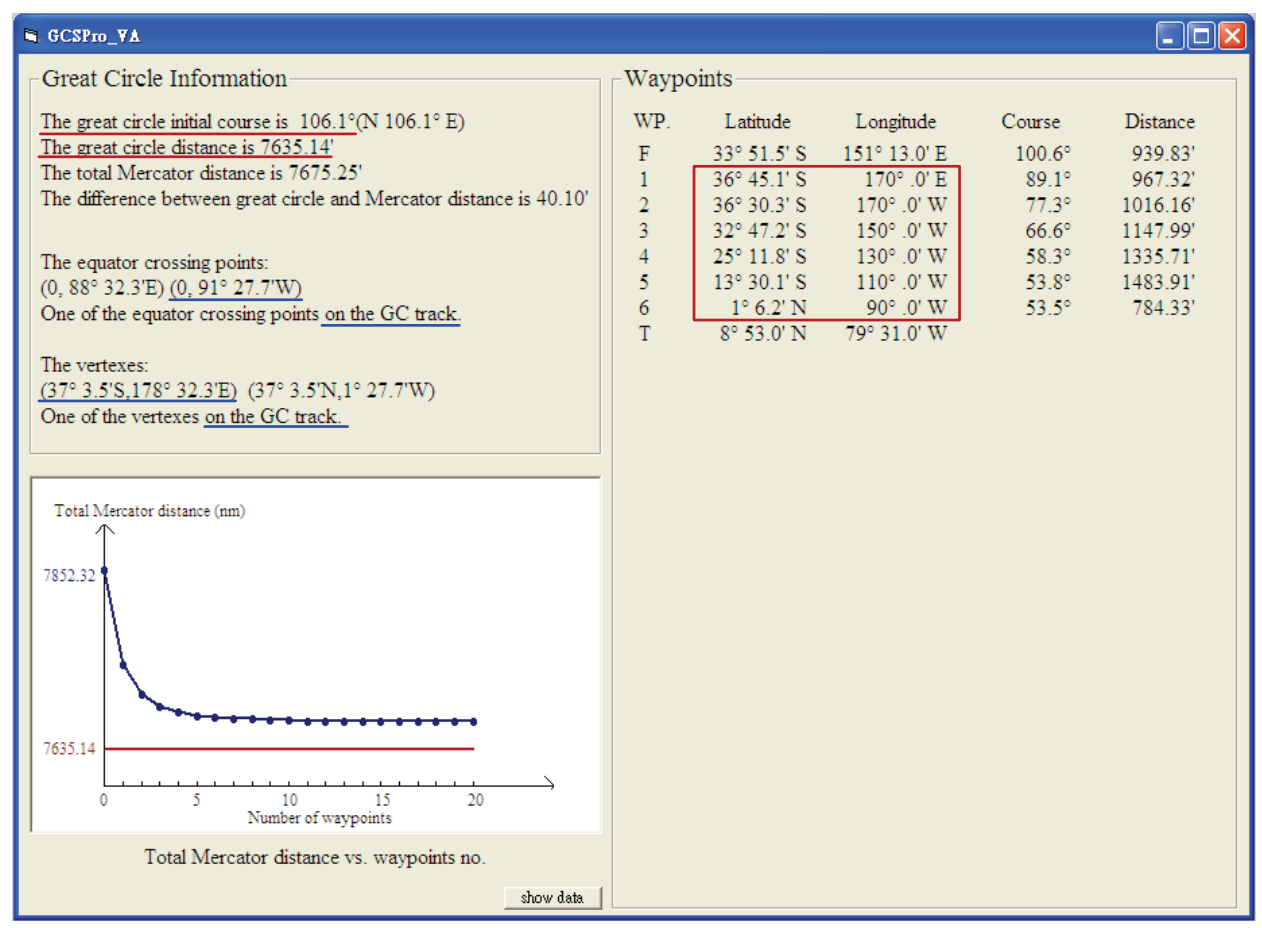

Fig. 4. Results of condition 2 by running the GCTPro_VA program in example 2

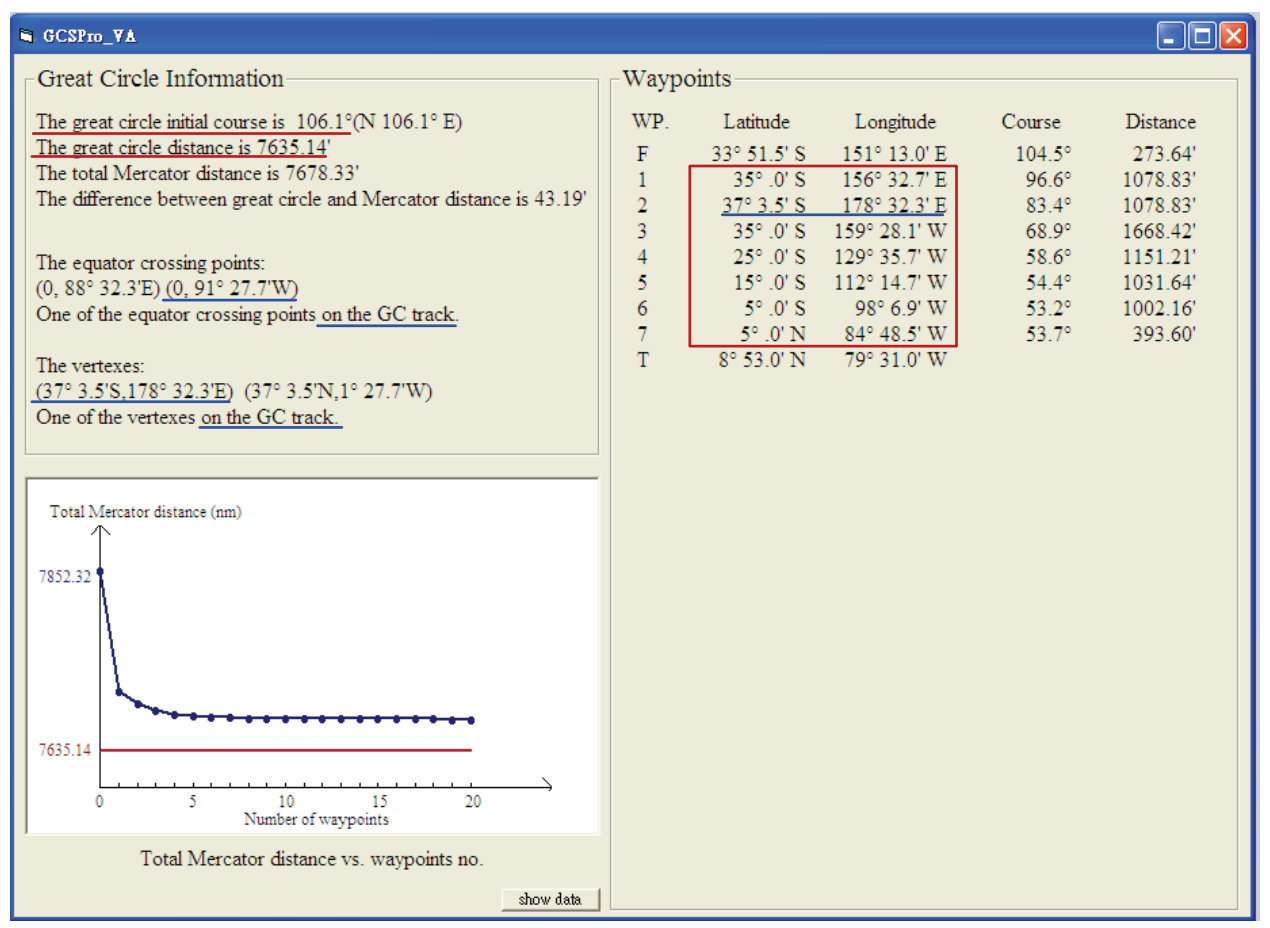

Fig. 5. Results of condition 3 by running the GCTPro_VA program in example 2

under all given initial conditions successfully. Because the mathematical properties of the geometry and algebra are both implied in the S-VA approach, the derivation process becomes more understandable and more straightforward as compared with those of conventional approaches. With the technique of transforming the Earth coordinates system into the Cartesian one and adopting the relative longitude concept, the derived governing equations by using the S-VA approach are simpler and clearer than those by the conventional approaches. Based on the S-VA approach, the GCTPro_VA program has been developed for friendly use of the navigator. Several benchmark examples are conducted to validate the proposed S-VA approach effectively and to verify the developed GCTPro_VA program successfully.

\section{ACKNOWLEDGEMENTS}

The author would like to express his thanks to Professor Jiang-Ren Chang for his valuable suggestions on this paper and Miss Pin-Fang Liu for drawing figures in this paper. In addition, the financial support from National Taiwan Ocean 
University under contract number: NTOU-103-001 is highly appreciated.

\section{NOMENCLATURE}

\begin{tabular}{|c|c|}
\hline $\mathrm{N}$ & North pole \\
\hline $\mathrm{S}$ & South pole \\
\hline $\mathrm{F}$ & the departure \\
\hline $\mathrm{T}$ & the destination \\
\hline$X$ & the waypoints on great circle track \\
\hline E & the equator crossing point \\
\hline$V$ & the vertex \\
\hline$L$ & the latitude \\
\hline$L_{\mathrm{F}}$ & the latitude of the departure \\
\hline$L_{\mathrm{T}}$ & the latitude of the destination \\
\hline$L_{X}$ & the latitude of the waypoints \\
\hline$L_{E}$ & the latitude of the equator crossing point \\
\hline$L_{V}$ & the latitude of the vertex \\
\hline$\lambda$ & the longitude \\
\hline$\lambda_{\mathrm{F}}$ & the longitude of the departure \\
\hline$\lambda_{\mathrm{T}}$ & the longitude of the destination \\
\hline$\lambda_{X}$ & the longitude of the waypoints \\
\hline$\lambda_{E}$ & the longitude of the equator crossing point \\
\hline$\lambda_{V}$ & the longitude of the vertex \\
\hline DLo & the difference of longitude from the departure to the destination \\
\hline$D L o_{\mathrm{F} X}$ & $\begin{array}{l}\text { the difference of longitude from the departure to the waypoints; a } \\
\text { dihedral angle in Fig. } 2\end{array}$ \\
\hline$D L o_{\mathrm{F} E}$ & $\begin{array}{l}\text { the difference of longitude from the departure to the equator } \\
\text { crossing point }\end{array}$ \\
\hline$D L o_{\mathrm{F} V}$ & the difference of longitude from the departure to the vertex \\
\hline$D$ & the great circle distance from the departure to the destination \\
\hline$D_{\mathrm{F} X}$ & the great circle distance from the departure to the waypoints \\
\hline$C$ & $\begin{array}{l}\text { the great circle initial course angle from the departure to the } \\
\text { destination; a dihedral angle in Fig. } 2\end{array}$ \\
\hline M & the meridional parts between the given latitude and the equator \\
\hline$a_{e}$ & the equatorial radius of the Earth, $a_{e}=3437.74677078 \mathrm{~nm}$ \\
\hline$e$ & the eccentricity of the Earth, $e=0.081819190842622$ from WGS- 84 \\
\hline$l$ & the difference of latitude between two adjacent waypoints \\
\hline dlo & the difference of longitude between two adjacent waypoints \\
\hline$\Delta M$ & the difference of meridional parts between two adjacent waypoints \\
\hline $\mathrm{cm}$ & the course angle in spherical Mercator sailings \\
\hline$d m$ & the distance in spherical Mercator sailings \\
\hline
\end{tabular}

\section{REFERENCES}

1. Bennett, G. G.: Practical Rhumb Line Calculations on the Spheroid. The Journal of Navigation, 49(1), pp. 112-119, 1996.

2. Bowditch, N.: American Practical Navigator. Volume 2, DMAH/TC, Washington, 1981.

3. Bowditch, N.: The American Practical Navigator. 2002 Bicentennial Edition, National Imagery and Mapping Agency, Maryland, 2002.

4. Chen, C. L.: New Computational Approaches for Solving the Great Circle Sailing and Astronomical Vessel Position. Ph.D. Dissertation, Department of Civil Engineering, National Taiwan University, Taipei, Taiwan, 2003.
5. Chen, C. L., Hsieh, T. H. and Hsu, T. P.: A Novel Approach to Solve the Great Circle Sailings Based on Rotation Transformation. Journal of Marine Science and Technology, 23(1), pp 13-20, 2015.

6. Chen, C. L., Hsu, T. P., and Chang, J. R.: A Novel Approach to Great Circle Sailings: The Great Circle Equation. The Journal of Navigation, 57(2), pp. 311-320, 2004.

7. Chen, C. L., Liu, P. F. and Gong, W. T.: A Simple Approach to Great Circle Sailing: The COFI Method. The Journal of Navigation, 67(3), pp. 403-418, 2014.

8. Clough-Smith, J. H.: An Introduction to Spherical Trigonometry. Brown, Son \& Ferguson, Ltd., Glasgow, 1966.

9. Cutler, T. J.: Dutton's Nautical Navigation. Fifteenth Edition, Naval Institute Press, Maryland, 2004.

10. Earle, M. A., Sphere to Spheroid Comparison. The Journal of Navigation, 59(3), pp. 491-496, 2006.

11. Greenberg, M. D.: Advanced Engineering Mathematics. Second Edition, Prentice-Hall International, Inc., 1998.

12. Holm, R. J.: Great Circle Waypoints for Inertial Equipped Aircraft. NAVIGATION, Journal of the Institute of Navigation, 19(2), pp. 191-194, 1972.

13. Jofeh, M. L.: The Analysis of Great-circle Tracks. The Journal of Navigation, 34(1), pp. 148-149, 1981.

14. Keys, G.: Practical Navigation by Calculator. Stanford Maritime, London, 1983.

15. Miller, A. R., Moskowitz, I. S. and Simmen, J.: Traveling on the Curved Earth. NAVIGATION, Journal of the Institute of Navigation, 38(1), pp. 71-78, 1991.

16. Nastro, V. and Tancredi, U.: Great Circle Navigation with Vectorial Methods. The Journal of Navigation, 63(3), pp. 557-563, 2010.

17. National Imagery and Mapping Agency (NIMA): Department of Defense World Geodetic System 1984: Its definition and relationship with local geodetic systems. Third Edition, Technical Report NIMA TR8350.2, 2000.

18. Royal Navy: Admiralty Manual of Navigation: The Principles of Navigation, Volume 1. 10th Edition. The Nautical Institute, London, 2008.

19. Spiegel, M. R., Lipschutz, S. and Spellman, D.: Vector analysis and an introduction to Tensor analysis. Second Edition, McGraw-Hill, 2009. 


\title{
CONTACT WITH THE AUTHOR
}

\author{
Chih-Li Chen, Ph.D.
}

Merchant Marine Department National Taiwan Ocean University 2 Pei-Ning Road, Keelung, 20224

$$
\text { TAIWAN }
$$

Email: clchen@mail.ntou.edu.tw 